\title{
LES SERVICES NUMÉRIQUES GRAND PUBLIC ET LEURS UTILISATEURS : TROIS APPROCHES SOCIOTECHNIQUES CONTEMPORAINES
}

Anna Jobin, Loïse Bilat

BSN Press | «A contrario »

2015/2 nº 22 | pages 107 à 113

ISSN 1660-7880

ISBN 9782940516544

Article disponible en ligne à l'adresse :

http://www.cairn.info/revue-a-contrario-2015-2-page-107.htm

\section{Pour citer cet article :}

Anna Jobin, Loïse Bilat, « Les services numériques grand public et leurs utilisateurs : trois approches sociotechniques contemporaines », A contrario 2015/2 ( $\left.\mathrm{n}^{\circ} 22\right)$, p. 107-113.

Distribution électronique Cairn.info pour BSN Press.

(C) BSN Press. Tous droits réservés pour tous pays.

La reproduction ou représentation de cet article, notamment par photocopie, n'est autorisée que dans les limites des conditions générales d'utilisation du site ou, le cas échéant, des conditions générales de la licence souscrite par votre établissement. Toute autre reproduction ou représentation, en tout ou partie, sous quelque forme et de quelque manière que ce soit, est interdite sauf accord préalable et écrit de l'éditeur, en dehors des cas prévus par la législation en vigueur en France. Il est précisé que son stockage dans une base de données est également interdit. 


\section{Les services numériques grand public et leurs utilisateurs: trois approches sociotechniques contemporaines}

ANNA JOBIN et LOÏSE BILAT

"If you are not paying for it, you're not the customer; you're the product being sold.»"

La première citation est celle d'un utilisateur du weblog Metafilter qui faisait remarquer que si vous ne payez pas pour quelque chose, ce n'est pas vous le client; vous êtes la marchandise. Ce slogan s'est vite popularisé et a été utilisé comme vérité révélatrice dans le cadre de discussions autour des plateformes numériques "gratuites ». Une vérité qui permet d'attirer l'attention sur la logique économique à laquelle sont soumises les plateformes numériques contemporaines les plus populaires, qui, à l'exception notable de Wikipedia, sont toutes des entreprises privées. Cette citation délégitime simultanément toute critique de la part des utilisateurs d'un service gratuit: un client peut se plaindre d'un service; une "marchandise" ne se plaint pas. De là, il n'y a qu'un pas jusqu’à la logique utilitariste: " si vous n'aimez pas, n'utilisez pas». Sous cet angle, l'interaction entre individus et plateformes numériques est considérée comme purement transactionnelle, présupposant des marchés transparents et des acteurs tant rationnels que bien informés. Dès lors, les individus choisissent librement si, et si oui à quelles conditions, ils sont d'accord de participer à ce type de transactions.

Outre de nombreuses critiques démontrant les limites réelles d'une telle conception idéologique, une étude empirique représentative récente confirme que la plupart des utilisateurs des services numériques ne se considèrent pas, en tant que tels, acteurs d'une transaction juste et transparente, bien au contraire. Le fait de partager

1 Contribution publié par l'utilisateur blue_beetle à 1:41 PM le 26 août 2010 sur le site Metafilter, disponible sous http://www. metafilter.com/95152/Userdriven-discontent\#3256046, consulté le 30 avril 2016. (activement) ou de laisser collecter (passivement) ses données personnelles par les services numériques est justifié par un sentiment de fatalité, d'impuissance envers ces services, 
plutôt que par le résultat d'une décision rationnelle (Turow et al. 2015). Les appareils multi-usages tels que les smartphones renforcent encore cette impuissance puisque ces supports et leurs applications communiquent entre elles, s'échangeant du contenu et des informations sans que l'utilisateur n'ait à les produire consciemment; pris dans un flux informationnel auquel il participe constamment, l'utilisateur n'a alors d'autre choix que d'éteindre tout appareil afin d'échapper à ce "sémiocapitalisme ${ }^{2}$ » qui tente d'accaparer son attention et réduit à néant sa sensibilité, dont les plateformes ne peuvent rien faire (Bifo 2013). Or, éteindre tout appareil signifie se priver de moyens de communication importants avec autrui. Ainsi, les " coûts" 108 d'une non-utilisation, pour parler en termes économiques, ne se calculent pas simplement à partir du fait d'« aimer», ou non, un appareil ou un service numérique.

La citation ci-dessus contient bien sûr des lacunes dans sa description de notre relation aux plateformes numériques contemporaines - ce n'est pas comme si, en payant, nous pouvions être sûrs de ne pas être la marchandise - mais le modèle économique de base qu'elle reflète, lui, est pertinent. Dans ce modèle, datant de bien avant les services numériques, l'entreprise en question sert simultanément deux (voire plusieurs) groupes d'utilisateurs distincts, dont l'existence des uns conditionne la présence des autres, créant ainsi un marché appelé bi- ou multi-face. ${ }^{3}$ A ce modèle correspondent notamment les médias dits traditionnels, financés au moins en partie par la publicité, mais ceci correspond également au modèle d'affaires des plateformes les plus utilisées comme Google et Facebook, dépendant à la fois des

2 «Le sémiocapitalisme est centré sur la création et la marchandisation des appareils techno-linguistiques (des images aux flux d'informations, des produits financiers aux logiciels) qui ont un caractère intrinsèquement semiotique et déterritorialisé. " (Bifo, 2013)

3 Pour mieux comprendre les marchés multifaces,voir URL: http://web.mit. edu/14.271/www/rochet_tirole.pdf, consulté le 12 mai 2016.

4 Jeffrey Hammerbacher, cité dans Ashlee Vance (2011), "This Tech Bubble Is Different", Bloomberg Businessweek, URL: http://www.fastcompany. com/3008436/takeaway/why-data-godjeffrey-hammerbacher-left-facebookfound-cloudera, consulté le 30 avril 2016. utilisateurs individuels et des annonceurs. La marchandise n'est cependant pas l'utilisateur à proprement parler mais son attention (comme pour les médias dits traditionnels, cf. Bermejo 2009), ses données (une nouveauté, cf. Lee 2011), ou les deux à la fois (cf. Citton 2014).

«The best minds of my generation are thinking about how to make people click ads.»4

Dans un entretien sur la crise économique, un mathématicien et ancien employé de Facebook regrette que ses pairs soient occupés à réfléchir 
sur les moyens de faire cliquer les gens sur des pubs. Cette citation reprend le poème désenchanté de Ginsberg, emblème de la Beat Generation aussi caractéristique de la Californie que la Silicon Valley, Howl («I saw the best minds of my generation destroyed by madness...", 1956). Ici, ce ne sont plus la guerre froide, le conservatisme et les stupéfiants qui menacent la société en s'attaquant à la santé mentale de sa jeunesse mais le marché du travail qui la tarit. Cette deuxième citation souligne le fait que la publicité en ligne occupe une place centrale dans l'économie numérique et, par extension, l'économie tout court (pour en savoir plus sur cet avènement cf. Crain 2014). Cette description traduit une vision beaucoup plus sombre de la relation entre les services numériques et leurs utilisateurs, une vision où l'indépendance et la marge de manœuvre de ces derniers est beaucoup plus restreinte. D’après elle, une personne peut être manipulée, amenée à faire quelque chose, le but déclaré étant de la "faire cliquer" sur des pubs. L’interaction entre individus et plateformes numériques n'apparaît plus du tout sous l'angle uniquement transactionnel mais est teintée d'enjeux, notamment de pouvoir et d'argent.

La vision optimiste d'un internet collaboratif qui rend la société plus égalitaire en donnant davantage d'autonomie aux individus cède ainsi la place à une certaine désillusion à l'encontre de ce qui apparait comme une perte de sérendipité et une illusion démocratique. Avec l'ascension progressive d'acteurs monopolistiques (Yahoo rachète GeoCities en 2002 et Flickr en 2005, Google rachète Youtube en 2006, Facebook rachète Instagram en 2012 etc.), cette désillusion n'est pas infondée. Agrégeant de grands nombres d'utilisateurs grand public - et, ainsi, leurs créations numériques et publications (user-generated content) - et imposant leurs standards techniques, les services numériques grand public se transforment en plateformes (Helmond 2015), remplaçant le web de la navigation hypertexte ouverte par un écosystème fermé (walled garden). Et ce qui au départ semblait une réalisation parfaite d'un média «many-to-many" s'est avérée, de plus en plus, structurellement identique aux médias de masse «one-to-many» (cf. Gunthert 2009).

Il n'est pas étonnant que la publicité en ligne soit une pierre angulaire de ces plateformes numériques, vu que les utilisateurs leur apportent de la valeur monétaire. Si un individu produit ou divulgue des données, cela permet au final un meilleur ciblage publicitaire; que cela cible la personne dont proviennent les données ou, comme souvent, d'autres personnes. Même par sa simple attention, sa paire d'yeux, dirigée pour quelques instants vers une pub, un individu enrichit le service numérique qu'il utilise. L'ensemble des différents modes de production de valeur 
par les utilisateurs, plus ou moins directs, est parfois abordé sous le nom de « digital labor" (Scholz 2012, Cardon et Casilli 2015). Un travail rarement reconnu en tant que tel, qui brouille les frontières entre production et consommation (à ce sujet cf. par exemple Ritzer et Jurgensson 2010, et leur terme de "prosommation"). Bien que les utilisateurs y participent en principe de manière volontaire, il ne faut pas perdre de vue l'asymétrie du pouvoir entre d'un côté les individus et de l'autre les plateformes numériques à but lucratif (Scholz 2016; pour une configuration analogue dans l’industrie du jeu de hasard cf. Schüll 2014).

"We don't need you to type at all. We know where you are. We know where you've been. We can more or less $[k]$ now what you're thinking about. ${ }^{5}$

À l'époque PDG de Google, Eric Schmidt déclare dans un entretien que Google n'a pas besoin que vous utilisiez votre clavier car l'entreprise sait où vous êtes, où vous étiez, et plus ou moins ce que vous êtes en train de penser. Si connaître l'emplacement d'une personne s'explique dans la plupart des cas de manière assez banale par les traces numériques géolocalisées qu'elle génère en utilisant un appareil connecté, savoir ce que quelqu'un est en train de penser mobilise une tout autre logique. Une logique reposant sur au moins deux hypothèses: la première présuppose que la pensée et sa forme d'expression constituent une seule et même chose. C'est en effet «seulement» à notre comportement (clicks, temps de lecture, mouvements des doigts ou de la souris etc.) et à nos mots exprimés que les services numériques ont accès. Croire pouvoir en déduire nos pensées relève d'une conception extrêmement limitée de l'esprit humain - il est cependant probable que faire le lien avec l'esprit n’est pas jugé nécessaire pour Google: une personne peut de manière skinnerienne être définie par son comportement et le langage accessible. Son « esprit » est ainsi transformable en données mesurables et modélisables.

La deuxième hypothèse part donc du principe qu'une personne est, de fait, modélisable, ce qui entraine des conséquences déjà visibles dans notre quotidien

5 Eric Schmidt, cité dans Derek Thompson (2010), "Google's CEO: "The Laws Are Written by Lobbyists" ", The Atlantic, URL: http:/| www.theatlantic.com/technology/ archive/2010/10/googles-ceo-thelaws-are-written-by-lobbyists/63908/, consulté le 30 avril 2016. connecté. Les inférences basées sur le profilage et ses données précédentes, croisées et comparées avec celles d'autres personnes, permettent de prédire son comportement actuel et futur comme ses pensées. Inutile de préciser que cette vision de l'individu ne considère plus du tout ce dernier comme un acteur 
libre, mais comme un pion dont la trajectoire est déjà déterminée - et mieux il est profilé, meilleures seront les prédictions. L’interaction avec les services numériques n'est à ce titre qu'une occasion parmi d'autres de récolter des données et tester des modèles de profilage. C'est d'ailleurs dans cette activité de profilage que le marketing en ligne possède des intérêts communs avec la surveillance étatique (Vaidhyanathan et Bulock 2014, cf. également Zimmer 2010 et Zuboff 2015). Le profilage sert in fine au ciblage, qu'il s'agisse d'afficher la bonne publicité au meilleur moment ou de prédire, et donc de prévenir, un comportement illicite. Ce que l'on pourrait nommer notre "nouvelle identité algorithmique " (Cheney-Lipold 2011) influence désormais de manière importante notre vie, ceci indépendamment de la question de savoir si sa catégorisation correspond à celle de notre identité vécue ou non.

L'impact de ce profilage ne se situe pas seulement au niveau individuel loin s'en faut; comme les logiques du ciblage publicitaire, les algorithmes de catégorisation, les autocomplétions et les contenus suggérés s'appliquent également aux contenus médiatiques de manière générale. Les informations, elles aussi, risquent de devenir hyper-personnalisées, résultant en une absence d'espace médiatique commun, partagé, ce qui constitue une menace potentielle pour la démocratie (Couldry et Turow 2014). 


\section{Références}

BERARDI [Bifo] Franco (2013), «Média-activisme revisité», Multitudes.

URL: http://www.multitudes.net/media-activisme-revisite/, consulté le 8 mai 2016 BERM EJo Fernando (2009), « Audience Manufacture in Historical Perspective: From Broadcasting to Google », New Media \& Society, vol. 11, N ${ }^{\circ} 1-2$, pp. 133-154.

CARDON Dominique et Antonio CASILLI (2015), Qu'est-ce quele digital labor?, Bry-surMarne, INA.

CHENEY-LIPOLD John (2011), "A New Algorithmic Identity. Soft Biopolitics and the Modulation of Control», Theory, Culture \& Society, vol. 28, $\mathrm{N}^{\circ}$ 6, pp. 164-81.

Cit ton Yves (2014), Pour une écologie de l'attention, Paris, Seuil.

COULDRY Nick et Joseph TUROW (2014), "Advertising, Big Data and the Clearance of the Public Realm: Marketers' New Approaches to the Content Subsidy ", International Journal of Communication, $\mathrm{N}^{\circ}$ 8, pp.1710-1726.

CRAIN Matthew (2014), «Financial Markets and Online Advertising: Reevaluating the Dotcom Investment Bubble», Information, Communication \& Society, vol. 17, $\mathrm{N}^{\circ} 3$, pp. 371-384.

GUNTHERT André (2009), “L’image partagée. Comment Internet a changé l'économie des images ", Études photographiques, $\mathrm{N}^{\circ} 24$, pp.182-209.

HELmond Anne (2015), «The Platformization of the Web: Making Web Data Platform Ready ", Social Media + Society, vol. 1, $\mathrm{N}^{\circ} 2$.

LEE Micky (2011), «Google Ads and the Blindspot Debate», Media, Culture \& Society, vol. $33, \mathrm{~N}^{\circ} 3$, pp. 433-447.

RITZer George et Nathan JURGenson (2010), «Production, Consumption, Prosumption. The Nature of Capitalism in the Age of the Digital "prosumer" ", Journal of Consumer Culture, vol. 10, $\mathrm{N}^{\circ}{ }^{1}$, pp. 13-36.

scHOLz Trebor (2012), Digital Labor: The Internet as Playground and Factory, Abingdon, New York, Routledge.

scholz Trebor (2016), Uberworked and Underpaid, Cambridge, Malden, Polity.

sCHÜLL Natasha (2014), Addiction by Design: Machine Gambling in Las Vegas, Princeton University Press.

TUROW Joseph (2015), The Tradeoff Fallacy: How Marketers Are Misrepresenting American Consumers and Opening Them Up to Exploitation, Philadelphia.

URL: https://www.asc.upenn.edu/sites/default/files/TradeoffFallacy_1.pdf, consulté le 30 avril 2016.

VAIDHYANATHAN Siva et Chris BULOCK (2014), «Knowledge and Dignity in the Era of "Big Data" ", The Serials Librarian, vol. 66, N ${ }^{\circ}$ 1-4, pp. 49-64. 
ZIM MER Michael (2010 [2008]), «The Gaze of the Perfect Search Engine: Google as an Infrastructure of Dataveillance ", in Web Search Multidisciplinary Perspectives, A. Spink et M. Zimmer (dir.), Berlin, Springer.

zUBoff Shoshana (2015), «Big Other: Surveillance Capitalism and the Prospects of an Information Civilization ", Journal of Information Technology, vol. 30, $\mathrm{N}^{\circ}$ 1, pp.75-89. 